\title{
PROYECTO GARCÍA
}

\section{La construcción de la Ciudad de la Costa.}

\author{
Gabriela Secco Terra \\ Arquitecta. Máster Urbanismo, DUOT. UPC
}

\section{RESUMEN}

Se analiza el proceso de urbanización de la Ciudad de la Costa, complejo y creciente territorio metropolitano montevideano, que a la vez es la segunda ciudad más importante de Uruguay. Entendido siempre como un proceso espontáneo y sin planificación de formación del territorio, se sugiere una mirada al mismo como un proyecto de suelo global que se realiza en un siglo, donde la agrimensura y la forestación adquieren crucial importancia. El proyecto se inicia con la adquisición de un gran campo por parte de un empresario que comienza a utilizar la forestación como instrumento de recuperación y cualificación de tierras pantanosas. Una primera partición de la tierra marca las principales trazas en el territorio, y un sucesivo proceso de fraccionamiento del suelo rural con visión urbanizadora, primero motivado por un reparto equitativo entre los descendientes de Doroteo García, pero luego por un proyecto urbanizador basado en la cultura de sol y playa y la especulación inmobiliaria, define los principales componentes de este proyecto.

Palabras clave: Ciudad de la Costa, balnearios, fraccionamientos, agrimensor. 


\section{ID_INVESTIGACIONES}

\section{ABSTRACT}

This article analyses the urbanization process of the Ciudad de la Costa, a complex and growing metropolitan territory in Montevideo and the second largest city of Uruguay. Always understood as a spontaneous configuration process and an unplanned territory, a view of a global land project is suggested, completed in one century and in which the surveying and afforestation acquires crucial importance. The project begins with the acquisition of a large field by a businessman who starts using forestry as a tool for recovery and qualification of wetlands. A first land partition defines the main traces in the territory, and it is followed by a process of rural land fragmentation with an urban vision: first motivated by an equal distribution among the descendants of Doroteo Garcia, but then by an urbanizing project based in the sun and beach culture and real estate speculation. This defines the main components of this project.

Key words: Ciudad de la Costa, seaside villages, parcelling, land surveyor.

\section{LA CIUDAD DE LA COSTA}

La construcción de la Ciudad de la Costa se caracteriza por ser desestructurada, producto del crecimiento espontáneo producido por la sucesión de particiones autónomas de suelo rural fraccionado por la iniciativa privada. Existe dentro de este territorio sin orden aparente una homogeneidad relativa en el conjunto, provocada por la suma de mecanismos y geometrías que se suceden y que le otorgan características tales que la diferencian de otros territorios. Esta investigación estudia los orígenes y procesos que se dan en este territorio, para poner en valor estos elementos característicos con el fin de establecer criterios de intervención de la zona. Defendemos que todos estos elementos constituyen un único proyecto de suelo global y no solo fragmentos concebidos individualmente. Éste se desarrolla a lo largo de un siglo y es un 


\section{ID_INVESTIGACIONES}

modelo de organización de ciudad, es decir que el territorio urbano resultante es fruto de un interesante proceso de fraccionamiento de suelo concretado mediante la relevante técnica de agrimensura y forestación. El proyecto de suelo consiste en la adecuación de un territorio para ser urbanizado mediante la forestación, la definición de una trama vial jerarquizada (rutas nacionales y departamentales), el establecimiento de un sistema de espacios libres (Bañado de Carrasco, Parque Nacional de Carrasco) y la creación de un parcelario coherente, conformado por piezas diferentes según su relación con las vías, las áreas costeras o las productivas. El artículo presenta las características generales del proyecto en todas sus fases, complementándolo con el análisis de un caso particular y la aproximación tentativa de su desarrollo en todo el territorio.

La Ciudad de la Costa se encuentra en el departamento de Canelones, al límite Este de Montevideo, zona actualmente muy dinámica debido a sus condiciones ventajosas de accesibilidad, cercanía al centro, y centralidad propia que han

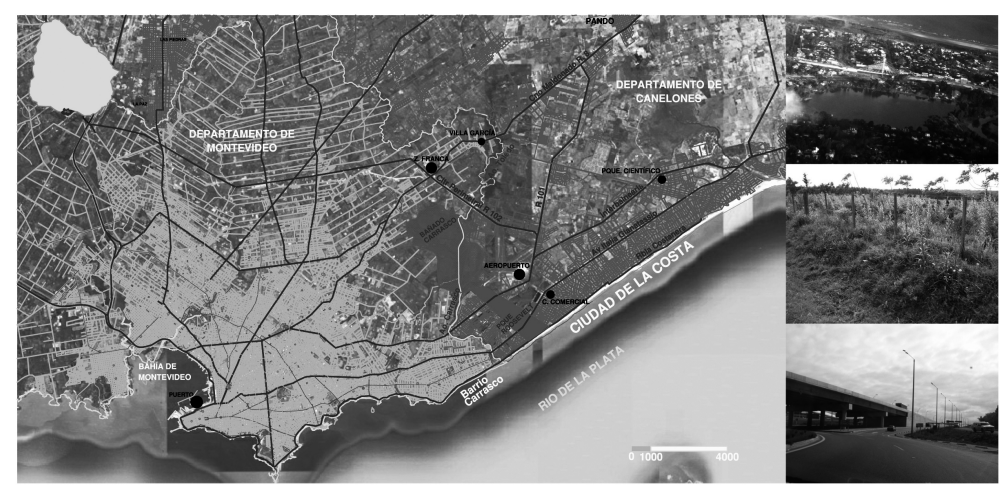

Ubicación. Fuente: Elaboración propia. Google Earth 


\section{ID_INVESTIGACIONES}

atraído emprendimientos comerciales, industriales y residenciales. Recibe la denominación de ciudad en 1994, aumenta su población en un 80 por ciento en los últimos veinte años ${ }^{1}$ y se afirma tiene el crecimiento demográfico más grande de América Latina en este período ${ }^{2}$. Más asombrosa es esta afirmación si constatamos que la población uruguaya posee un crecimiento demográfico despreciable en éste período, por lo que este incremento se debe esencialmente a desplazamientos provocados por la continua expansión montevideana. Este territorio concentra además de la población, grandes actividades de afluencia nacional. Lo atraviesan dos de las rutas nacionales más importantes del país, que en esta zona definen la puerta de entrada a Montevideo y su puerto comercial. Son por esta misma razón corredores de servicios comerciales e industriales donde se encuentra una importante zona franca, y el aeropuerto internacional de Carrasco. Allí se da una superposición de dinámicas a diferentes escalas y estructuras diversas. Un alto valor productivo, ambiental y paisajístico converge con un gran desarrollo industrial, de servicios y grandes infraestructuras.

La estructura física de la zona está compuesta básicamente por la franja costera del Río de la Plata con un sistema de dunas, donde para estabilizarlas la forestación tuvo un papel muy importante; por manchones de área rural productiva y un sistema de humedales conexos y de alto valor ambiental denominado Bañado de Carrasco y Cuenca del Arroyo Carrasco, que desemboca en el Parque F.D. Roosevelt, ex Parque Nacional de Carrasco. A partir de la década de 1950 la Ciudad de la Costa se gesta como una suma de balnearios, pueblos de veraneo de la clase media montevideana, que reposan sobre los 16 kilómetros de playa, con una estructura de ciudad jardín.

\footnotetext{
${ }^{1}$ Instituto Nacional de Estadísticas. www.ine.gub.uy

2 SELLANES, Enrique. 2001. Guía de la Ciudad de la Costa.
} 


\section{ID INVESTIGACIONES}

La Ciudad de la Costa y sus inmediaciones conforman lo que antiguamente era el campo de Doroteo García. Una extensa estancia de 8 mil hectáreas es adquirida por este empresario, hijo de españoles, en 1835, y es en este momento cuando comienza el proceso urbanizador del área. Destaca el papel emprendedor que juega la familia García, tanto el propietario inicial de las tierras, Doroteo García; como su señora e hijos, letrados y responsables de diversos emprendimientos estatales y sociales. En una época en que el país apenas se esta construyendo, los vemos plasmar sus nuevas ideas y visiones civilizadoras en estas tierras.

Una primera división se produce entre los herederos de Doroteo García; sus siete hijos y esposa, y son éstos límites los que precisamente determinan las primeras trazas viales del territorio. Este proceso de división continúa entre los hijos de los hijos, y sucesivamente, por lo que la causa motora del fraccionamiento del suelo en una primera etapa, que va hasta mediados del siglo $\mathrm{XX}$, es la descendencia familiar.

La continua fragmentación de las tierras entre descendientes García se transforma, en una segunda etapa, en un negocio inmobiliario, al adquirir estas tierras valor por su cercanía a la costa. Las mismas dejan de estar ligadas al sector rural productivo para entrar al servicio de la cultura de veraneo, y segunda residencia a modo de válvula escape de la capital. Tanto en el primer momento, donde el fraccionamiento se rige por la equidad de las herencias como en el segundo, por la especulación y la venta; los ingenieros forestales y los ingenieros agrimensores adquieren un rol fundamental en los procesos. Son acondicionadores del lugar, intérpretes de las leyes, y ejecutores de la urbanización. 

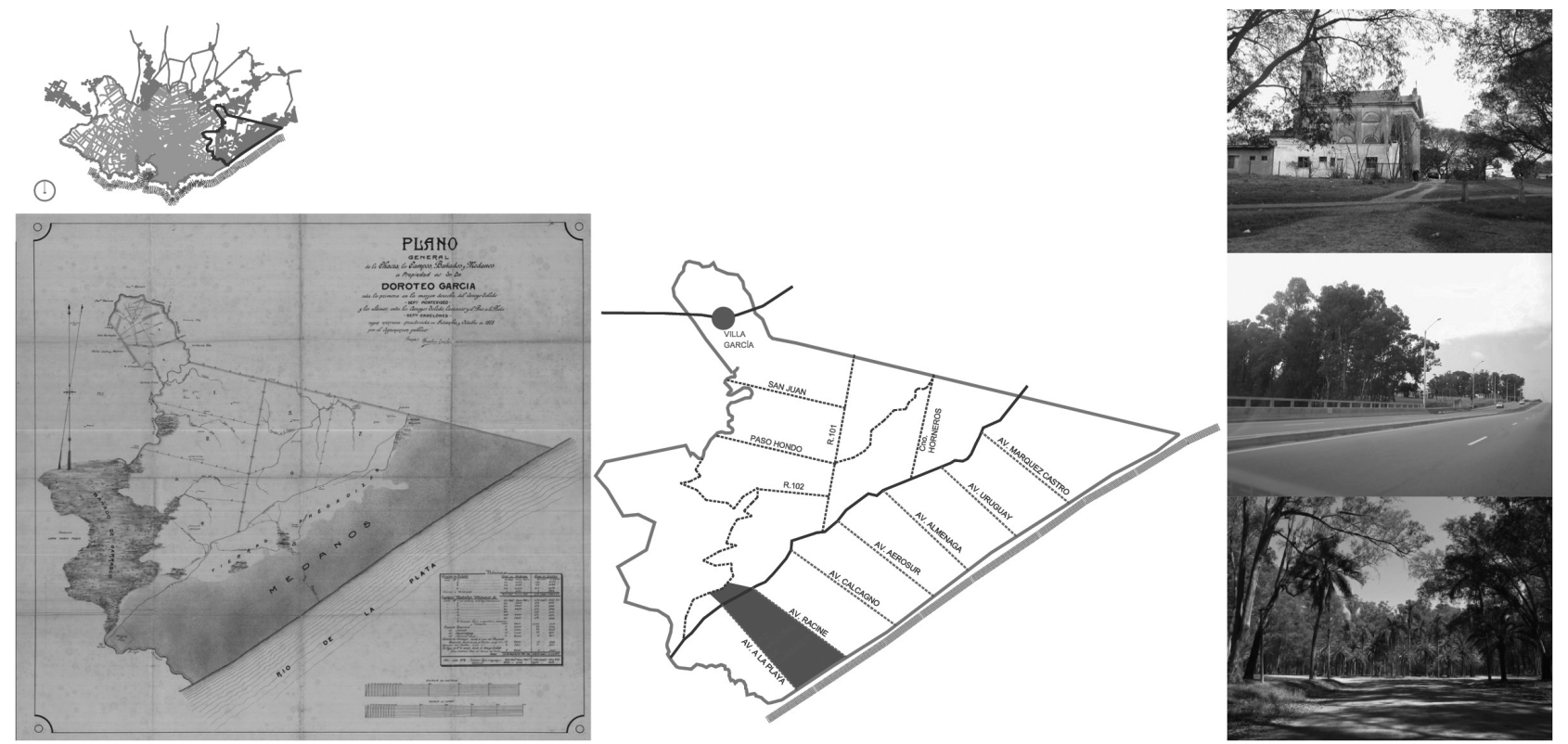

Primer fraccionamiento. Principales vías. Situación actual de: Villa García. Ruta 101.

Parque F.D.Roosevelt. Plano Fridolin Quinke 1878. Fuente: Elaboración propia.

\section{LA CONSTRUCCIÓN DE UN PAÍS}

Es importante destacar el papel que juega Doroteo García como personaje preocupado por el progreso del país y la productividad del territorio. Doroteo García Arguibel (1806-1885), hijo de Ildefonso García, comerciante español, y de Teresa de Arguibel, argentina, se caracteriza por ser un hombre emprendedor que vela por el progreso del país. Se educa en Inglaterra, y, entre otras cosas es nombrado Ministro de Hacienda de Uruguay en 1835. En 1843, 


\section{ID_INVESTIGACIONES}

durante la Guerra Grande de Uruguay $^{3}$, se traslada a Buenos Aires con su familia debido a reparos ideológicos. De regreso, mientras se suprime la esclavitud en Uruguay y aparecen los alambrados como herramienta de división entre propiedades rurales como símbolo del orden, prosigue con sus acciones.

Dedica muchos días de intensa tarea a la colonización en épocas en que la República necesitaba muchos brazos ${ }^{4}$. En 1858 funda la Sociedad Agrícola del Rosario Oriental con 34 accionistas, sociedad que se encarga de la localización de las familias de inmigrantes en tierras agrícolas. Con el fin de poblar el país con europeos conocedores de agricultura, la Sociedad compra tierras y las subdivide para venderlas a los colonos en cuotas y paralelamente se ocupa de fundar los pueblos impulsando la creación de la iglesia y la plaza central. En nombre de esta sociedad Doroteo García funda el pueblo de La Paz el 17 de octubre de 1858 y el 25 de abril de 1862 funda la Colonia Suiza denominándose Nueva Helvecia, con los Piamonteses que se trasladan desde el departamento de Florida. La Plaza de la Villa La Paz en Colonia Valdense lleva hoy su nombre. "...le di palabra de venderles trescientas sesenta cuadras de terreno sobre Sarandí Chico en el sitio designado, reservándome decidir a su tiempo la ubicación...Algo es ya para el porvenir de la Colonia, empezar poblando bajo buenas condiciones...He decidido pasar a verificar la división de las chacras para los colonos y la designación del futuro Pueblo que voy a situar en la tapera de Figueredo, a distancia de cuatro cuadras del Rosario y $13 \mathrm{del}$ excelente Puerto que hemos encontrado..."

\footnotetext{
${ }^{3}$ La Guerra Grande (1839-1851) es un conflicto político de lucha entre nacionalistas y gauchos, frente a otro grupo más afín con la instauración del orden civilizador. Contó con la intervención de Argentina,

Brasil, Francia e Inglaterra.
${ }^{4}$ Directamente ligado a la idea de "Gobernar es poblar" de J.B. Alberdi. Personaje intelectual relevante de la historia rioplatense del siglo XIX. Durante la Guerra grande de Uruguay desde Montevideo apoyó, en favor de los colorados, la intervención francesa contra el gobierno de Rosas. ALBERDI, Juan Bautista. 1852. Bases y puntos de partida para la organización política de la República Argentina. Edición digital a partir de la edición de Francisco Cruz, Buenos Aires, La Cultura Argentina, 1915

partir de la edición de Francisco Cruz, Buenos Aires, La Cultura Argentina, 1915.
Extractos del cuaderno de viaje de Doroteo Garcia. WIRTH, Juan Carlos F. 1980. Génesis de la colonia agrícola suiza Nueva Helvecia. Ed. Ministerio de Educación y Cultura.
} 


\section{ID INVESTIGACIONES}

En 1871 Doroteo García impulsa la fundación de la Asociación Rural de Uruguay, hasta hoy activa. Sus hijos van adquiriendo roles importantes en la formación de la República, como miembros del tribunal Supremo de Justicia, redactores del código penal y civil, o de la reforma de la enseñanza, directores de la Compañía de ferrocarriles, y alentadores de la educación popular.

En el reparto de tierras hecho por el gobierno español por intermedio de Pedro Millán, se adjudica a Esteban de Ledesma el campo de Toledo-Carrasco en suerte de estancia. El gobernador Intendente de Buenos Aires, Francisco de Paula Sáenz, la vende a Melchor de Viana, quien a su vez en 1835 la vende a Doroteo García. Desde 1838 Doroteo García se dedica a los trabajos agropecuarios en su estanzuela, que puebla con inmigrantes de Canarias y de Galicia, los primeros inmigrantes que llegan a Uruguay. Instaura industrias agrícolas para el cultivo del gusano de seda, la fabricación de alcohol y vinagre de fruta, y el cuidado de las abejas.

Doroteo García deja plasmado su afán de hacer más productivo su territorio mediante numerosas operaciones forestales innovadoras. Si bien la parte de Toledo (al Norte de la estanzuela) es altamente productiva, el resto se compone prácticamente de dunas y tierras anegadizas. Por esta misma razón en 1868 , Doroteo García se presenta ante el poder ejecutivo de la República solicitando salidas del domino fiscal las tierras, bañados y médanos comprendidos dentro de los límites de sus títulos, "quedando éstos libres y al abrigo de las pretensiones del fisco"; estas tierras improductivas.

Doroteo García, junto con otros interesados como Thomas Tomkinson?, introduce eucaliptos malanoxylon desde Australia y otras especies inexistentes

\footnotetext{
${ }^{6}$ Escritura Hijuela de la Sucesión del Doctor Carlos García Acevedo. Escribano José M. Durán Guani.

1936. Su antigua quinta es hoy parque Municipal de Montevideo.
} 

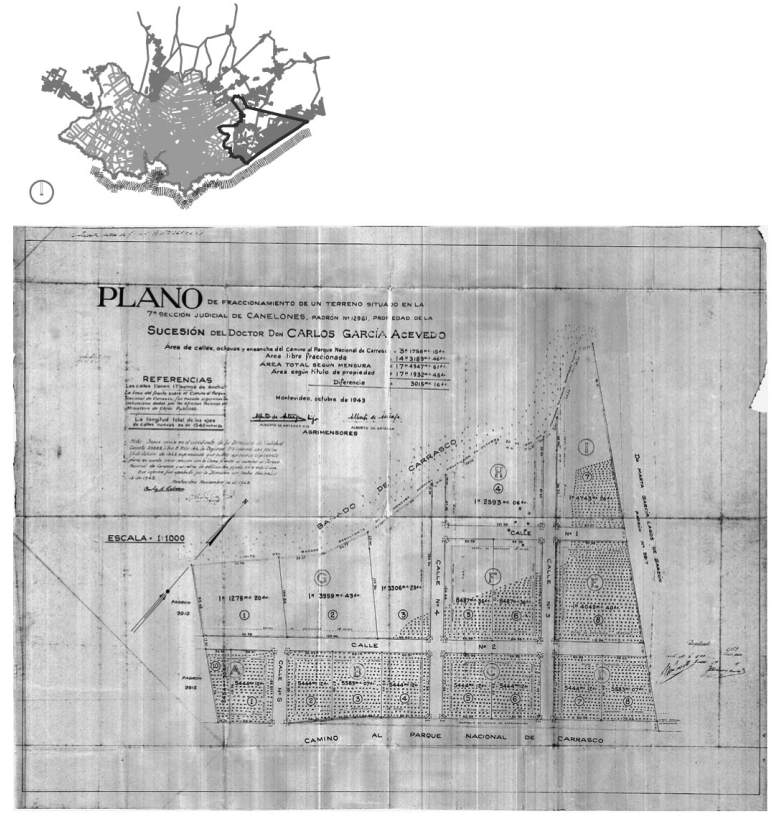
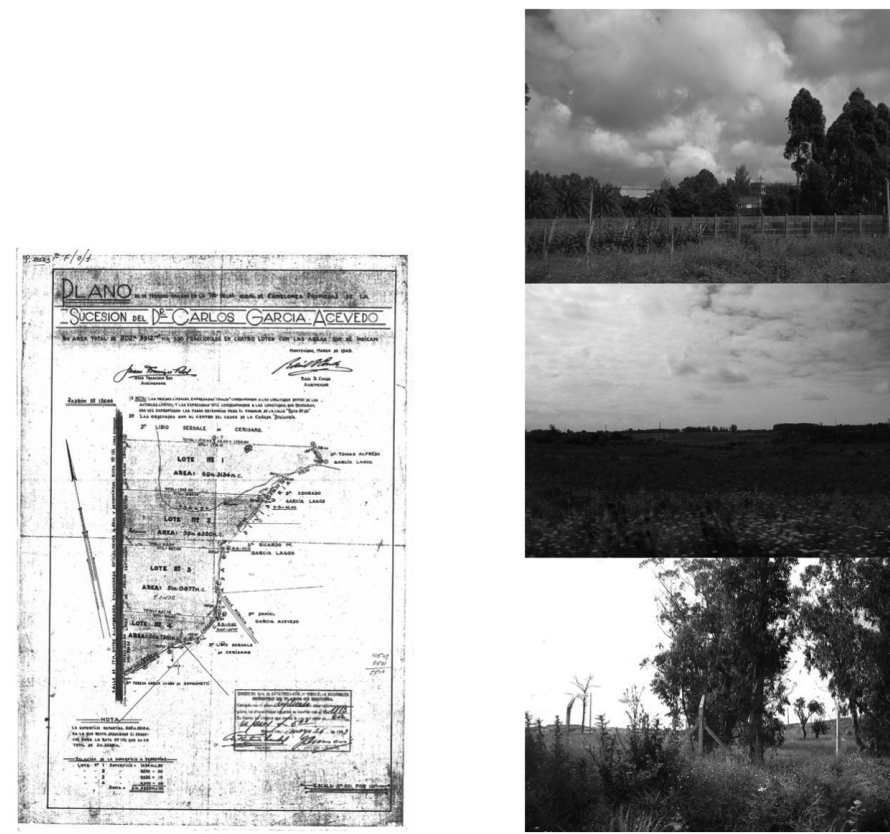

Fraccionamientos diversos de 1943. Situación actual de Chacras y quintas. Sucesión Carlos García Acevedo. Ing. Agmr. A. de Arteaga hijo, A.de Arteaga. Ings. Agmrs. J. Francisco Ros, R. Conde

en el territorio de la Banda Oriental. A la manera europea comienza numerosas plantaciones de diversas especies novedosas que prometen un buen desarrollo en estas tierras. En su chacra de Toledo planta eucaliptos y álamos mayoritariamente, y al Sur experimenta con pinos para fijar las dunas e intentar desecar el bañado. ${ }^{8}$

${ }^{8}$ Cabe aclarar que entonces no se percibía el alto riesgo ambiental que tal operación proporcionaba, ya que no existía tal conciencia ecológica, sino altamente productiva. 


\section{ID I N VESTIGACIONES}

\section{EL PROYECTO DE SUELO}

\subsection{Las primeras trazas. Preparación del terreno.}

El agrimensor Fridolin Quincke realiza en 1878 la medición de los campos de Doroteo García. En 1885, después de la muerte de Doroteo García dichas fracciones se reparten entre sus herederos, 7 hijos y su señora Carolina Lagos.

En esta primera división se determinan tres zonas con características diferentes entre sí, a ser subdivididas. La primera zona es la chacra del Arroyo Toledo, de pequeña extensión, pero muy productiva, que se divide en 4 secciones, todas ellas destinadas a su esposa, salvo la cuarta destinada a su hijo mayor. La segunda es la de los campos de Bañados, de gran extensión,medianamente productiva pero más inaccesible e inhóspita por el viento y la arena que volaba hasta allí. Ésta se divide en 7 partes para cada uno de los hijos. Por último, la zona de médanos y tierras anegadizas, improductiva en ese entonces, que se le entrega a Carolina Lagos. Antes de morir Carolina Lagos en 1906, esta última fracción de médanos se subdivide en 8 partes proporcionales, destinadas a sus hijos, salvo una que queda como continuación del Bañado de Carrasco, y que se convierte en lo que es hoy el parque Roosevelt. Todos estos primeros límites entre las divisiones de los campos coinciden con lo que hoy son las principales rutas nacionales, caminos departamentales y principales avenidas de la Ciudad de la Costa.

Como uno de los primeros actos urbanizadores del territorio, Carolina Lagos funda en 1889 el pueblo Villa García. La cuarta fracción de la chacra de Toledo es donada por su hijo mayor Ildefonso García Lagos en favor de su madre con el objetivo de contribuir a la voluntad de ésta de fundar un pueblo en el que ella misma hace edificar una capilla en memoria de Doroteo García. La parte donada se divide en 6 manzanas iguales destinadas a la edificación y una plaza. Villa García se funda con todas las características normales de un 


\section{ID_INVESTIGACIONES}

pueblo, con sus manzanas de cien por cien metros en torno al camino principal, y la plaza e iglesia como estructuradores centrales. El eje principal es el Camino Maldonado, importante vía que une el puerto de Montevideo con el interior del país. La villa es atravesada por el Arroyo Toledo, que hace de límite de los departamentos de Montevideo y Canelones. En 1908 se fundan una escuela, y una policlínica de salud.

Dentro de la sucesión de los García-Lagos figura en 1917 la donación al Estado del Bañado de Carrasco y el Parque Nacional de Carrasco, correspondientes a la franja de los médanos que limita con el mar. Siguiendo con la voluntad de Doroteo García, el primer objetivo de esta donación es la desecación del bañado por parte del Estado. Al ser tierras improductivas y difíciles, se pretende que el Estado mejore su condición a través de plantaciones. No obstante, se declara en la donación como un proyecto de parque público de recreo, lo que demuestra el interés de la familia García por realizar una vez más un aporte social a la nación. "Mis hermanos y yo estamos prontos a contribuir a la realización del proyecto de desecación y parque público donando al Estado toda la parte del Bañado de Carrasco que nos pertenece..., y además una zona de trescientas cincuenta hectáreas de terreno contiguo para extender hasta la costa del Río de la Plata el parque proyectado dando así a la obra mayores atractivos para el público y permitiendo ir haciendo el parque en esta zona mientras se realizan los trabajos de desecación en el bañado",

En la donación se impone la condición de que si en diez años no se desecan los terrenos cedidos, éstos se deben devolver. Es por esta razón que para el ${ }^{9}$ Escritura de Donación del Parque Nacional de Carrasco y Bañados de Carrasco. Escribano Juan José
Belo. 1917 

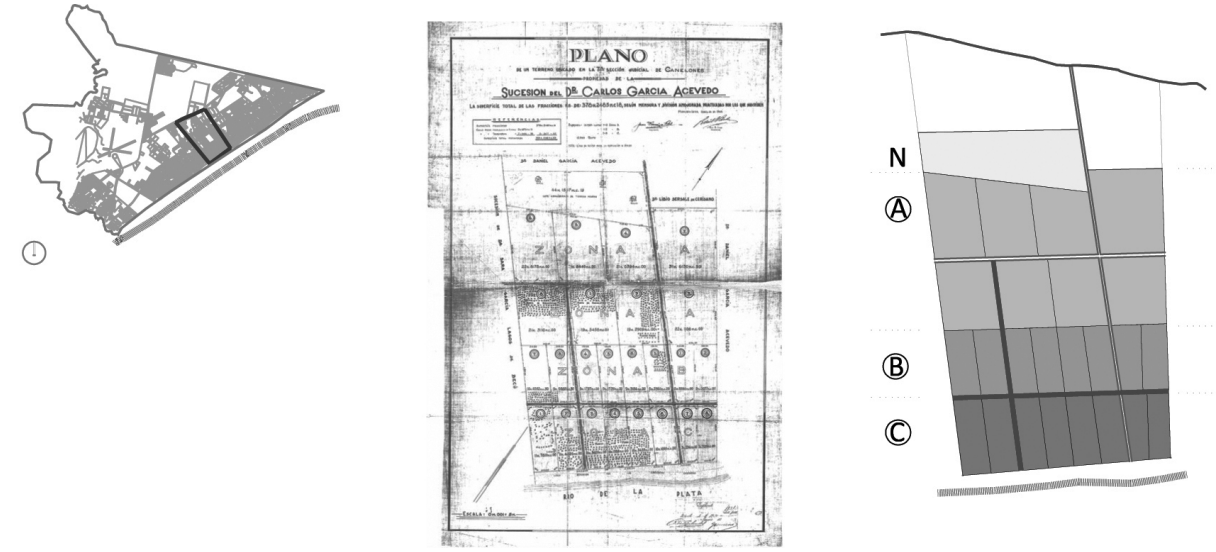

Caso de estudio. Situación del parcelario en 1943. Ings Agmrs. J.F. Ros, R. B. Conde

diseño se acude rápidamente al paisajista francés Carlos Racine, ${ }^{10}$ para que comience con la tarea de proyectar el parque público. Una veintena de hombres en medio de una naturaleza inhóspita y de difícil acceso realiza la transformación de las dunas y tierras anegadizas mediante la creación de caminos y la forestación con eucaliptos, sauces, álamos, robles, acacias, gran variedad de pinos y una herradura de palmeras al centro. Carlos Racine realiza posteriormente la administración del Parque Nacional de Carrasco y quien le sucede es su alumno el Ing. Gabriel Caldevilla, quien se desempeña después como paisajista urbanizador en la creación de los balnearios.

Otra condición es que se destine a parque público de libre acceso. Esto supone que los descendientes de los hermanos García-Lagos en diversas ocasiones tienen que reclamar al Estado el mal uso del parque y bañado, como en la época de la tenencia militar, y en la actualidad. En febrero de 2011 se realiza
1943

\begin{tabular}{|c|c|c|c|}
\hline \multicolumn{4}{|l|}{ dc. Carlos Garcl } \\
\hline \multirow{2}{*}{\multicolumn{4}{|c|}{$\begin{array}{l}\text { fraccionamiento: CHACRAS } \\
\text { técnica: EQUIDAD de las PARTES_Zonas } \\
\text { GEOMETRIA REGULAR } \\
\text { cesión: } 2 \text { nuevos CAMINOS DE ACCESO } \\
\text { (Ley de Vialidad 1865: ancho } 17 \mathrm{~m} \text { ) }\end{array}$}} \\
\hline & & & \\
\hline T.NEGRAS & & 44Has & (Inutilizables) \\
\hline $\begin{array}{l}\text { ZONAA } \\
\text { (Interbalnearia) }\end{array}$ & $\times 8$ trace & ${ }_{21 \text { Has }}$ & Eucaliptus \\
\hline $\begin{array}{l}\text { ZONAB } \\
\text { (cerca mar) }\end{array}$ & $\times 8$ fracc & 9Has & $\begin{array}{l}\text { Pinios } \\
\text { Eucaliptus }\end{array}$ \\
\hline $\begin{array}{l}\text { ZONAC) } \\
\text { (primera linea } m\end{array}$ & & & \\
\hline
\end{tabular}




\section{ID_INVESTIGACIONES}

un llamado de re-proyectación del parque con la voluntad por parte del Plan estratégico de Ordenamiento Territorial de la Ciudad de la Costa de reposicionar al Parque F.D. Roosevelt a nivel metropolitano, dada su estratégica situación. Algunas propuestas con fines de lucro y acceso restringido, como un estadio, causan nuevamente polémicas.

3.2 La sucesión García. Trayectoria de los fraccionamientos (1917-1947).

Los procesos de división de las tierras continúan con la misma lógica que el primer fraccionamiento. Los hermanos García Lagos dividen cada uno sus tierras entre sus respectivos hijos, que a su vez la reparten entre sus herederos. La causa motora del fraccionamiento del suelo en este territorio es hasta los años 50 la descendencia familiar. El proceder técnico es similar, y en esta época rige sobretodo la equidad de las partes. Cada territorio se divide en zonas de similares características, y cada una de ellas en el número de herederos correspondiente. Las zonas en este caso no se distinguen según su productividad, como ocurre en la primera división, porque las partes a fraccionar ya son zonas homogéneas en cuanto a su productividad. La división en zonas viene determinada ahora por su situación, es decir, si están más o menos próximas a una vía importante, el bañado o la costa. Esto quiere decir que se diferencian según el valor de la tierra en relación a su potencial urbanizable, y no a la producción. Cada una de estas zonas pasa entonces a subdividirse en el número de herederos, coincidente en casi todos los casos con el número de hijos.

La unidad de loteo en esta etapa ya no son campos, sino que corresponde a chacras de entre 20 y 5 has. En algunos casos y donde el suelo pasa a tener categoría de suburbano en lugar de rural, se realizan fraccionamientos posteriores de quintas, unidades de loteo menores de 5 has. De los bordes de todas estas particiones nace la trama de caminos de comunicación interna existente. Cada vez que se fracciona un terreno, el ingeniero agrimensor debe 


\section{ID_INVESTIGACIONES}

prever, especificándolo claramente, los caminos de acceso a todas las particiones. En principio se crean nuevas vías solamente por razones de accesibilidad para llegar a todos los campos o chacras, pero ya con las quintas se generan manzanas con calles en sus cuatro lados.

La definición de la propiedad va tomando cada vez más importancia en el país y aparecen nuevos organismos de regulación, entre ellos las Oficinas de Catastro y Mensura y el Ministerio de Obras Públicas, con su Dirección de Vialidad. La aplicación de las leyes provenientes de los organismos emergentes por parte de los fraccionadores define características fundamentales. El Ministerio de Obras Públicas determina el ancho de las nuevas calles, lo que establece el carácter de las mismas, y cuánta superficie deben ceder al Estado los propietarios de las parcelas para infraestructura vial. El propietario, además de dador de la vía, queda responsable en muchos casos de su ejecución, y de su manutención. Además se concretan los retiros de edificación según la importancia de las vías.

Dada una topografía tan llana, las particiones están basadas en una geometría regular. Ésta se define como principal herramienta estructuradora del territorio en esta etapa. Los principales factores perturbadores de la regularidad de los trazados son los bañados o cauces de agua presentes en el terreno. Por el contrario, las vías existentes son definitorias del sentido de los límites a establecer, ya que los nuevos límites se trazan perpendiculares y paralelos a las vías existentes.

Los descendientes de los García siguen con la prometedora tarea emprendida por su antecesor de plantar árboles. Acondicionar el territorio, estabilizar las dunas y desecar los bañados parece crucial, sobre todo cuando ya se empiezan a vislumbrar las primeras experiencias urbanizadoras exitosas e inspiradoras de los años 30 , como el balneario de Carrasco, limítrofe con la 


\section{ID_INVESTIGACIONES}

Ciudad de la Costa del lado de Montevideo. Juan María Pérez, empresario, coetáneo de Doroteo García y socio de éste en más de un emprendimiento, adquiere la tierra donde se situará el balneario de Carrasco en 1834. Más adelante la tierra se divide entre los herederos, y después de muchos años de ser prácticamente inaccesible, el proyecto se realiza en 1907 por otro empresario precursor, el Dr. Alfredo Arocena. Con él se constituye en 1912 la S.A. Balneario Carrasco y comienza la venta de solares para segunda residencia. Quien proyecta el plano urbanístico inicial del balneario es el arquitecto paisajista francés Carlos Thays ${ }^{11}$, a quien se le encomienda el proyecto de una ciudad jardín que se resuelve con trazados curvilíneos y un gran hotel que estructura la urbanización. Es destacable la adecuación de la naturaleza, al crear un paisaje forestal profundo y rellenar los bañados con arena y piedra.

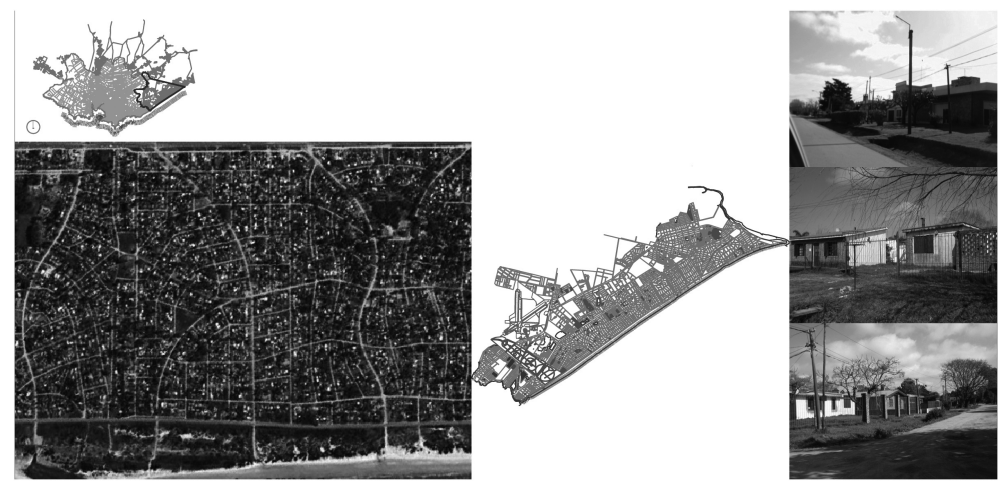

Parcelario Ciudad de la Costa. Situación actual de balnearios. Fuente: Elaboración propia. Base Plano del Parcelario D.N.Catastro, Google Earth.

${ }^{11}$ Carlos Thays (1849-1934): Paisajista francés creador de numerosos parques argentinos como el Jardín Botánico y el Parque 3 de febrero en Buenos Aires. Diseñador de la Plaza Independencia y colaborador en el diseño del Parque Rodó en Montevideo 


\section{ID_INVESTIGACIONES}

3.3 La Fiebre Fraccionadora ${ }^{12}$. Punto de inflexión (1947-1980).

A mediados del siglo XX se produce una intensificación de la actividad fraccionadora en la Ciudad de la Costa, ocasionada por el aumento del valor de estas tierras. Las parcelaciones tienen ahora como objetivo principal la compraventa. Nos encontramos en este período con un Estado desarrollado y adelantado en cuanto a sus emprendimientos ${ }^{13}$. Entre estos esta el desarrollo de las vías Nacionales, y desde los años 30, una voluntad explícita en fomentar el turismo, mayoritariamente costero. En 1947 aparece el aeropuerto. Un año antes, se define por primera vez una ley para los Centros Poblados del país, hecho que influye directamente en la concreción de este territorio. Las imposiciones que se realizan a las nuevas urbanizaciones tienen menos control fuera de la capital uruguaya, por lo que la Ciudad de la Costa se pone rápidamente en la mira al estar tan cerca de Montevideo.

El proyecto de García toma entonces vigor en el sector costero y comienza la construcción de los balnearios. En este momento las pautas de actuación provienen de un solo grupo emprendedor con una visión unitaria del sector y objetivos urbanizadores definidos. Prima la voluntad de crear barrios residenciales de alta categoría, referenciándose a otras experiencias internacionales. Para ello se establecen una seria de controles edificatorios, se implantan servicios, se crean y adecuan vías responsables de la homogeneidad apreciable hoy en día. Se forma un conjunto de balnearios similares entre sí, con un trazado ciertamente particular y sin duda inspirado en el modelo del vecino barrio de Carrasco y en los propósitos de la ciudad jardín.

\footnotetext{
${ }^{12}$ Término utilizado en MARTíNEZ GUARINO, Ramón. Libro blanco del área metropolitana | Canelones | Montevideo | San José. Editorial Agenda Metropolitana, Montevideo, Uruguay. 2007.

${ }^{13}$ Luis Batlle Berres (1897-1964): presidente de Uruguay en 1947. En esta época se denominó al país "la Suiza de América" por su estabilidad democrática y modélico poder ejecutivo, la fuerza de la clase media Suiza de América" por su estabilidad democrática y modélico poder ejecutivo, la fuerza de la clase media
y el alto nivel cultural y de alfabetización logrado. El auge económico de este período es el resultado del y el alto nivel cultural y de alfabetización logrado. El auge económico de este período es el resultado del
país beneficiado por la Segunda Guerra Mundial y la Guerra de Corea, la nacionalización de empresas como la británica de ferrocarriles, y el alto crecimiento de la industria.
} 


\section{ID_INVESTIGACIONES}

Los principios de ordenación manejados responden a una voluntad explícita de respetar la topografía existente, con tal de favorecer los desagües hacia el mar. Además, pensados como barrios jardín, estos fraccionamientos propician apreciaciones paisajísticas de recorridos y visuales.

El grupo emprendedor esta integrado por un par de empresarios precursores que compra tierras en estos médanos y comienza a forestarlas, dados los buenos resultados de los experimentos ya muy avanzados de los García. A su vez, este equipo de empresarios que se denomina MAR S.A. es contratado para realizar la forestación en muchas otras tierras aledañas, algunas aún pertenecientes a los descendientes García, que poco a poco van vendiéndolas a precios ínfimos para la realización de fraccionamientos en solares. La empresa Urbanizadora MAR S.A. es responsable de la creación de gran parte de los balnearios de la Ciudad de la Costa (Solymar, Lagomar, Colinas de Solymar, Médanos de Solymar). Está compuesta por técnicos especializados y asociada a una agencia inmobiliaria encargada de las ventas, Héctor Zunino.

El ingeniero agrónomo forestal Gabriel Caldevilla ${ }^{14}$ procede a determinar los criterios generales en cuanto a plantaciones y trazados viales, respetando la topografía del lugar de 2 metros de desnivel desde la vía principal (Ruta Interbalnearia) y la costa. Los criterios manejados intentan imitar la naturaleza y preservar las tierras de la erosión por medio de calles sinuosas. Previo acopio de plantaciones en viveros, el procedimiento para forestar consiste en realizar barreras de vegetación seca para contener la duna y parar el viento. Se planta una barrera de acacias longifolias que fija la duna por sus raíces grandes y posee buena adaptación a los vientos salobres. Una vez crecidas las acacias, se plantan los pinos en retícula que quedan protegidos del viento por éstas; luego sólo queda combatir la liebre, las hormigas y los incendios. La masa de

${ }^{14}$ Gabriel Caldevilla: encargado a su vez de la conservación y cuidado del Parque Nacional de Carrasco por parte de Ministerio de Ganadería Agricultura y Pesca. Educado como paisajista en Michigan. 


\section{ID_INVESTIGACIONES}

plantación es muy densa, de 1000 plantas/ha, para asegurar un crecimiento acelerado del árbol en vertical. El árbol no se expande en horizontal por falta de espacio, y crece solamente hacia arriba buscando la luz.

Con este procedimiento se llega en 10 años 10 metros de altura, que corresponde a 200 metros de protección contra vientos y arena en horizontal. Los criterios generales definen además las áreas para la edificación, y los espacios libres, muchas veces coincidentes con tierras anegadizas, áreas muy inundadas, o con la presencia de algún curso de agua.

El ingeniero vial José P. Deangelis, realiza el replanteo de los caminos, para comenzar a construirlos. Acto seguido y ya con la estructura definida se procede al fraccionamiento y mensura de los lotes por parte de un agrimensor, Carlos Hughes. Una vez aprobado el fraccionamiento por parte de las autoridades se pone a la venta, muy promocionado y en cómodas cuotas. MAR S.A. se encarga de la realización de ciertos proyectos de equipamientos, y por medio del arquitecto Saint Romain establece recomendaciones para la construcción de las viviendas con el fin de conservar las características de barrios de categoría. Para construir rápidamente se ofrecen bonificaciones a los nuevos propietarios, tales como el proyecto entero de la casa, la ejecución de los pozos de desagüe, o inclusive ladrillos de regalo. MAR S.A. repite este esquema de ejecución en todos los balnearios, dado su éxito. Se realizan diversos equipamientos como el Club Hípico, el Parador Solymar, y pequeñas previsiones comerciales.

Se manejan dos criterios de fraccionamiento. Las tierras cercanas al mar se fraccionan en solares de 400 o 500 metros. Las manzanas en general intentan ser largas y estrechas, de esta manera se pueden realizar más solares en las manzanas próximas a la costa y así el comprador puede enunciar que se encuentra "a dos o tres cuadras del mar" y no a más. Por otro lado, en las tierras más alejadas, se realizan particiones de quintas de 1 a 5 has, donde se 


\section{ID_INVESTIGACIONES}

proponen actividades de cultivo, deporte ecuestre y otras. En general se promueven los solares en relación con los servicios existentes, y no solamente con el sol y la playa.

La urbanizadora MAR S.A. se encarga de realizar la caminería interna y mantenerla, y cuidar de las tierras de los incendios mediante guardabosques. Una vez alcanzado un determinado porcentaje de ventas, se retira. Por ello, el mantenimiento va empeorando paulatinamente. Aparecen vías principales que no conectan con las partes de duna y los caminos principales resultan realmente accesibles recién cuando empiezan a asfaltarse en 1959. A partir de los años 80 comienza un proceso de transformación de la Ciudad de la Costa ocasionado por el fenómeno de desplazamiento de los pobladores montevideanos. Se densifica y se convierte en una zona de primera residencia, ocasionando diversos problemas de ajustes de infraestructuras.
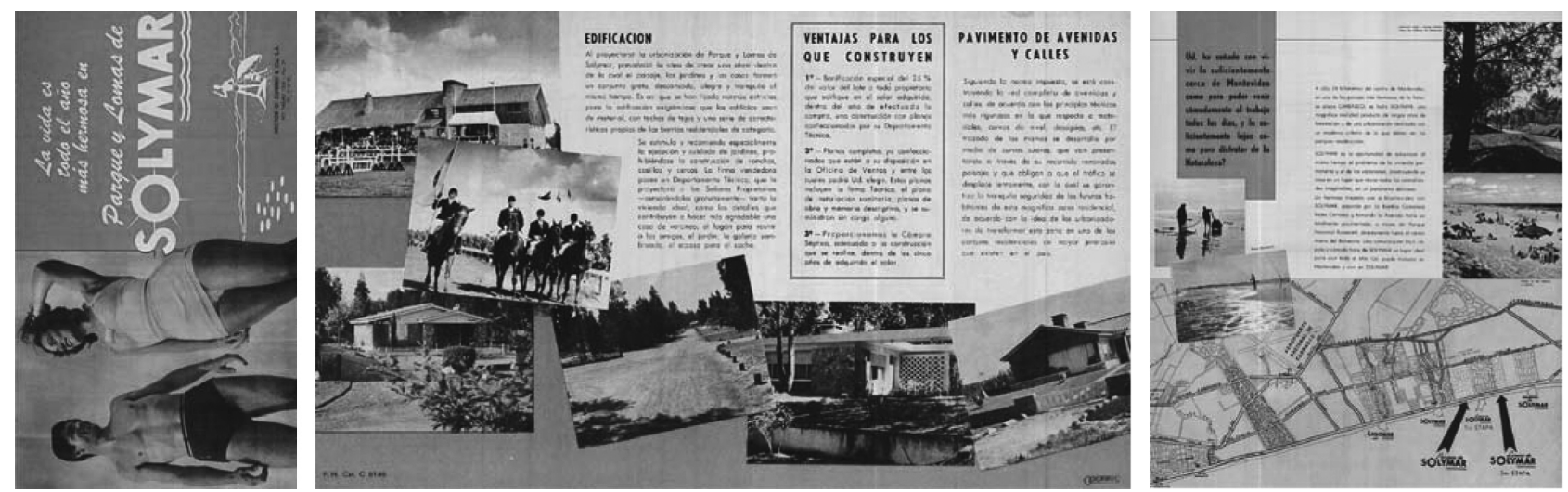

Folletería promocional de Solymar. HECTOR O. ZUNINO Y CIA .S.A. 


\section{ID_INVESTIGACIONES}

\subsection{La técnica}

La familia García ha constituido sin duda alguna una pieza fundamental para la construcción de este territorio. Doroteo García realiza emprendimientos forestales visionarios y sus hijos la donación del Parque Nacional de Carrasco y Bañados al Estado para su uso público. Posteriormente, hay un constante interés de los familiares por preservar las condiciones de donación originales de este gran parque metropolitano, velando para que no tuviese otros destinos en varios momentos de la historia. Por otra parte, cuatro generaciones realizan la sucesiva fragmentación de la tierra que constituyó la formación de prácticamente toda la trama vial existente en el área y la continuación del proceso forestal, que determina la forma base del territorio actual.

El ingeniero agrimensor es quien determina físicamente las distribuciones en el territorio mediante la medición, fraccionamiento y partición de las tierras y es el responsable del plano final de cualquier partición, por lo que tiene un rol crucial en la concreción de todo el territorio, y por ende en la tarea de hacer ciudad. El agrimensor es además técnico imprescindible para cualquier acto legal de repartición o venta, ya que para un visado o regularización de cualquier índole, desde reforma a urbanización, la ley uruguaya exige su firma.

Mediante el conocimiento de prácticas basadas principalmente en la geodesia, la aplicación legal, y la representación simbólica de situaciones territoriales según conclusiones obtenidas en trabajo de campo; se pueden establecer las características de su proceder que determinan la conformación de un tipo de ciudad y no otro. Es recién en 1888 que la facultad de Matemáticas, ex Facultad de Ingeniería, comienza a expedir los títulos de agrimensor, y en 1895 se crea la Dirección General de Catastro, que es el organismo encargado de la inscripción de los bienes. El proceder del agrimensor está ligado a la evolución de las leyes uruguayas a lo largo de todo el proceso; las técnicas de partición 

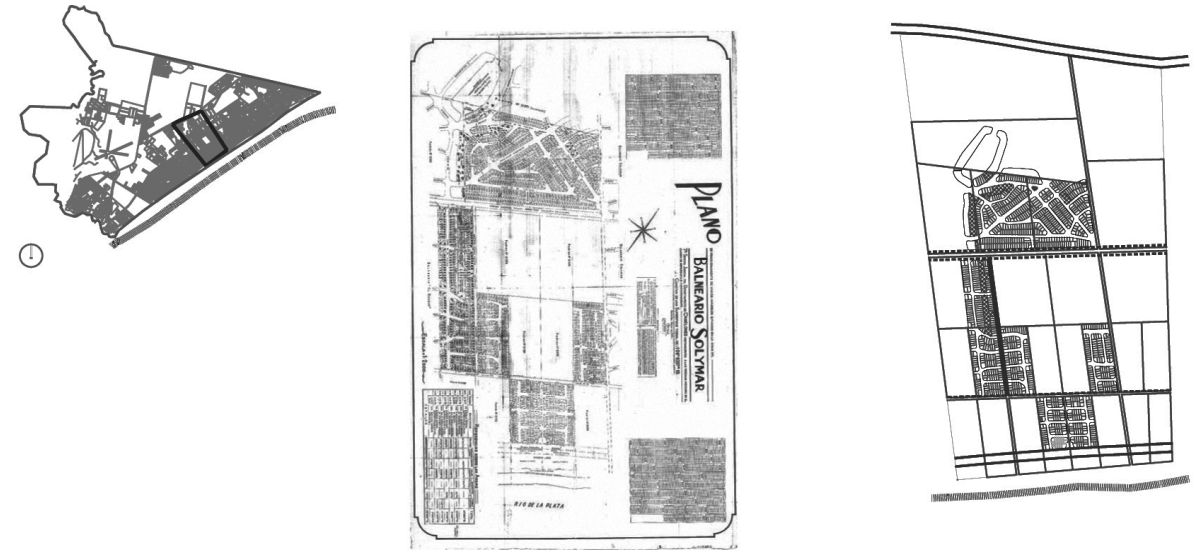

1956

Suc. Carlos Garcia Acevedo: SOLYMAR

fraccionamiento: SOLARES BALNEARIO técnica: VENTA INMOBILIARIA CURVAS TOPOGRAFÍA, RECORRIDO cesión: Ensanche vial Avda Italia de 27 a $60 \mathrm{~m}$. Nueva Calle 72 de 17 a $20 \mathrm{~m}$.

(Ley Calif. y Juridicc. de caminos 1946)

Caminería interna /E. Públicos/Equip.

MANZANA

SOLAR TIPO

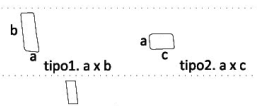

$300 \mathrm{~m} . / 15 . \mathrm{m}$ frente RETIROS $\quad 10 \mathrm{~m}$ Avdas $2 \mathrm{~m}$ bilateral $20 \%$ fondo

ESP.LIBRES

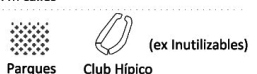

Caso de estudio. Situación del parcelario en 1956. Ing. Agmr. Carlos Hughes

evolucionan y se complejiza con ellas. En 1869 aparece la primera ley de vialidad, que determina que los nuevos caminos deben tener un ancho no menor a 17 metros. Durante el gobierno de José Batlle y Ordóñez ${ }^{15}$ la infraestructura nacional adquiere mucha importancia y se crea en 1907 el Ministerio de Obras Públicas y con él, la Dirección Nacional de Vialidad.

Posteriormente, en 1912 aparece la ley de expropiación de bienes raíces en plena planificación de las carreteras nacionales, y en 1946 la Ley de centros poblados que tiene muchísima influencia en la conformación de la Ciudad de la Costa. Estas exigencias, acatadas a rajatabla por los agrimensores, que a su vez son pocos los que trabajaron en esta zona, son garantes de la uniformidad de estos fraccionamientos.

${ }^{15}$ José Batlle y Ordóñez (1856-1929): dos presidencias 1903 y 1911 de Uruguay, con importantes reformas políticas y sociales; estatizaciones diversas, divorcio, voto de la mujer, separación del Estado y la Iglesia 


\section{ID_INVESTIGACIONES}

La figura del ingeniero forestal se ha encargado por otra parte de continuar con las iniciativas forestales de los García hasta la década del 50. Utiliza todo su conocimiento para ir creando una masa forestal adecuada para establecer los médanos y urbanizar la zona, teniendo como premisa el respeto de la topografía existente para facilitar los desagües y favorecer las visuales. La forestación habilita el desarrollo de la zona e impide la desaparición de la duna en su totalidad.

\subsection{Aproximación al modelo}

Las técnicas y mecanismos utilizados por los diversos personajes involucrados dotan al territorio de características comunes que transforman la supuesta espontaneidad y el crecimiento libre de esta zona, en un proyecto de suelo. Por primera vez se realiza la lectura histórica de este territorio peculiar, y encontrar su identidad puede ofrecer pautas para futuras actuaciones en un territorio de tales características. Al interpretar el proceso de su creación, se definen patrones que son comunes también a otros procesos urbanizadores costeros basados en el fraccionamiento del suelo rural uruguayo. En mayor o menor intensidad, el fraccionamiento del suelo rural con fines urbanizadores en la costa y su forestación masiva para el establecimiento del terreno, se ha producido en toda la extensión costera nacional.

Las fases del proyecto global de la Ciudad de la Costa y sus principales características quedan ejemplificadas mediante el estudio de una fracción particular; el caso de estudio. En ella se perciben las transformaciones según cada período y la técnica correspondiente. En la primera fase se determinan los límites que serán luego vías principales. Posteriormente, en una segunda fase, se distingue la geometría como reguladora de las parcelas y caminos. En la tercera fase, las parcelas geométricas se ven rellenadas con una trama de menor grano de solares y vías curvilíneas. Las manzanas creadas en esta etapa intentan quedarse, en la mayoría de los casos, indeterminadas en sus 
ID_INVESTIGACIONES

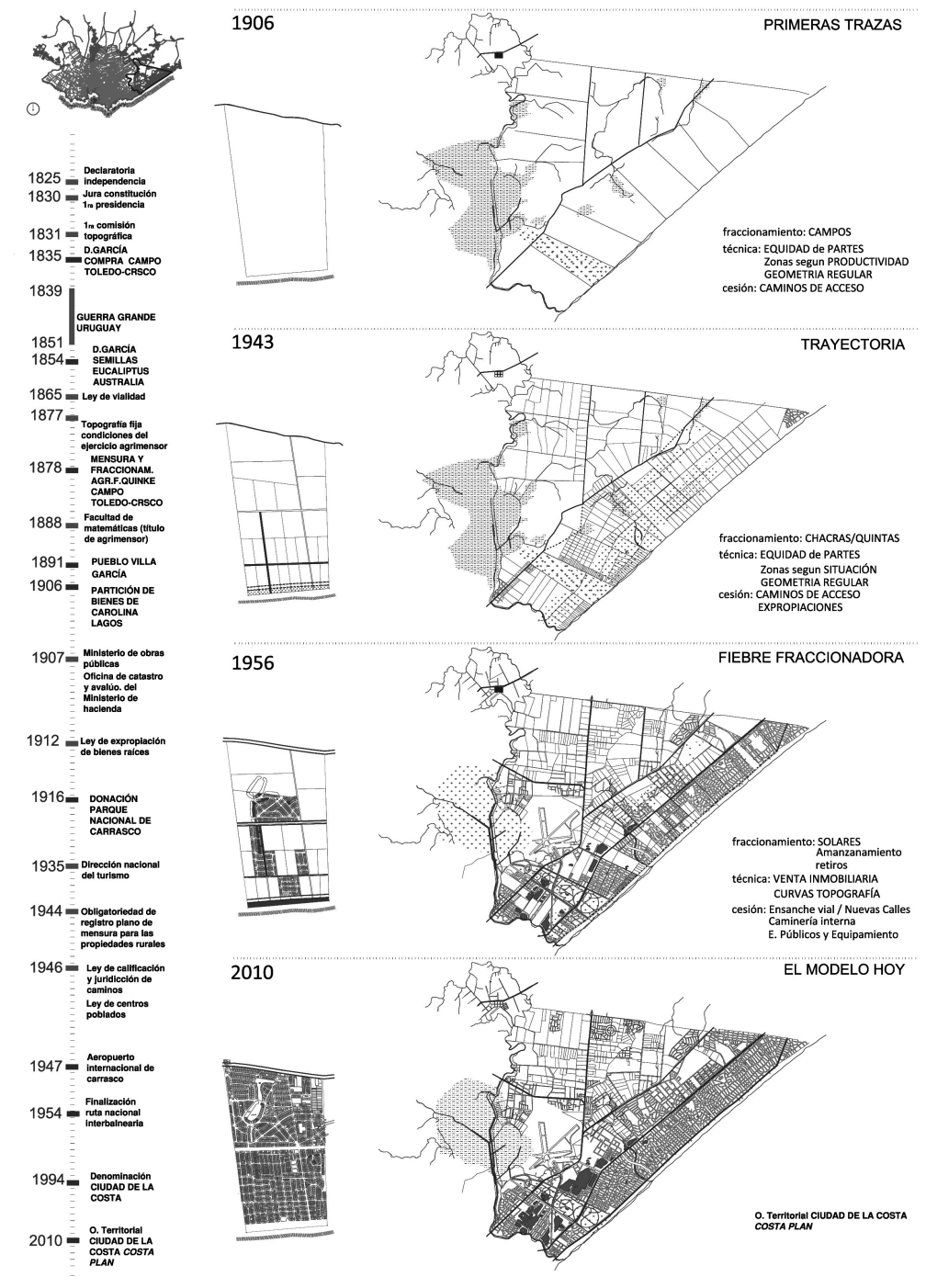

Proceso aproximado en todo el territorio y técnica de fraccionamientos según la época. Elaboración propia. Base planos varios. 


\section{ID_INVESTIGACIONES}

bordes para asegurar la continuación del trazado en los siguientes fraccionamientos. Las tres fases superpuestas son perceptibles en el parcelario actual como muestra el cuadro a continuación.

El proyecto ha sabido adaptarse a los sucesivos cambios de usos y necesidades de este territorio. La existencia de grandes campos y la producción rural extensiva da paso a la producción intensiva y construcciones de descanso; las chacras y las quintas. Éstas se han ido sustituyendo por balnearios en la zona costera, los cuales a su vez supieron densificarse y transformarse en ciudad de primera residencia. La primera etapa de urbanización representa hoy los grandes estructuradores de la Ciudad de la Costa, es decir, el parque metropolitano y las vías más importantes. La adecuación de la franja costera mediante la forestación posibilita el asentamiento residencial que se constituye con características muy apreciadas por un gran sector de la población montevideana. El continuo fraccionamiento del suelo en manos de un oficio en particular, que si bien evoluciona a lo largo de este siglo, mantiene una técnica determinada que permite ver el territorio como una única pieza de proyecto, coherente en sus resoluciones.

\section{GLOSARIO}

AGRIMENSOR: topógrafo.

ARROYO: corriente natural de agua que fluye con continuidad aunque con escaso caudal BALNEARIO: pueblo o barrio dedicado al veraneo, vacaciones, descanso, esparcimiento; baños de mar.

BAÑADO: humedal, terreno húmedo, inundado por aguas pluviales o por las de un río o laguna cercana (marisma).

CAMPO, ESTANCIA: hacienda de campo destinada al cultivo, generalmente a la ganadería. 
ID_INVESTIGACIONES

CHACRA: del quechua antiguo chajra; maizal. Granja. Predio rural (mayor a 5 has).

DEPARTAMENTO: parte del territorio del país sujeta a una autoridad administrativa municipal.

ESTANZUELA: diminutivo de estancia

FRACCIONAMIENTO: acto de partir el territorio.

GAUCHO: mestizo que en los siglos XVIII y XIX habitaba el Río de la Plata y Río Grande, buen jinete y diestro en los trabajos ganaderos, hombre de campo.

HIJUELA: documento notarial donde se reseñan los bienes que tocan en una partición a cada uno de los partícipes en el caudal que dejó un difunto.

MÉDANO: duna.

QUINTA: finca de recreo alejada de la ciudad. Huerta dedicada al cultivo de hortalizas. Predio Suburbano (hasta 5 has).

RUTA: carretera nacional, ancha, pavimentada y dispuesta para el tránsito de vehículos

\section{BIBLIOGRAFIA}

AA.VV. Revista de la Asociación Rural .189

AA.VV. 1942. Revista del Instituto Histórico y Geográfico del Uruguay, Volúmenes 1617.Ed. Inst. Histórico y Geográfico del Uruguay.

AA.VV 1907, 1908. Anales de la Universidad, Republica Oriental del Uruguay. Ed. Escuela Nacional de Artes y Oficios.

AA.VV. 2011. Proceso de ocupación del territorio. Facultad de Arquitectura.www.farq.edu.uy/estructura/servicios_docentes (03. 2011)

BARRÁN, J. Pedro. NAHUM, Benjamín. 1978. Historia rural del Uruguay Moderno: 18511885 (2v), Ediciones de la Banda Oriental. 
ID_I N VESTIG ACIONES

BARRIOS PINTOS, Anibal. 1971. Montevideo, los barrios. II., Ed. Daniel Aljanati. Montevideo.

COSTA PLAN.2010. Plan Estratégico Ordenamiento Territorial Ciudad de la Costa. Intendencia Municipal de Canelones.

Dirección Nacional de Catastro, Ministerio de economía y finanzas. www.catastro.gub.uy

Dirección Nacional de Topografia, Ministerio de Transporte y Obras Públicas. www.dntopografia.gub.uy

Entrevistas a: Ing. Agrónomo Carlos Colombino: Emprendimientos del Sr. Emilio Colombino, MAR.S.A. Ing. Forestal Gabriel Caldevilla (hijo): Procedimientos de las operaciones urbanizadoras de MAR.S.A. Pautas forestales y del sistema dunar. Trazado vial. Creación del Parque Roosevelt.

Facultad de Ingeniería. Universidad de la República. www.fing.edu.uy/ia

MARTÍNEZ GUARINO, Ramón. Libro blanco del área metropolitana | Canelones | Montevideo | San José. Editorial Agenda Metropolitana, Montevideo, Uruguay. 2007.

ODDONE J.A. 1966. La emigración europea al Río de La Plata, Montevideo, Ediciones de la Banda Oriental.

SERRA, Enric. 1995. Geometria i Projecte del sòl als origens de la Barcelona moderna. La Vila de Gracia. Col.lecció d'Arquitectura. U.P.C. Barcelona

WIRTH, Juan Carlos F. 1980. Génesis de la colonia agrícola suiza Nueva Helvecia. Ed. Ministerio de Educación y Cultura. 